\title{
Failure of cefoxitin and doxycycline to eradicate endometrial Mycoplasma genitalium and the consequence for clinical cure of pelvic inflammatory disease
}

\author{
C L Haggerty, ${ }^{1}$ P A Totten, ${ }^{2}$ S G Astete, ${ }^{2}$ S Lee, ${ }^{1}$ S L Hoferka, ${ }^{1}$ S F Kelsey, ${ }^{1}$ R B Ness ${ }^{1}$
}

${ }^{1}$ University of Pittsburgh, Pittsburgh, USA; ${ }^{2}$ Department of Medicine, Division of Infectious Diseases, University of Washington, Seattle, USA

Correspondence to: Catherine L Haggerty, University of Pittsburgh, Department of Epidemiology, 130 DeSoto Street, 516B Parran Hall, Pittsburgh, PA 15261; haggerty@pitt.edu

Accepted 9 April 2008 Published Online First 29 April 2008

\section{UNLOCKID}

This paper is freely available online under the BMJ Journals unlocked scheme, see http:// sti.bmi.com/info/unlocked.dtl

\begin{abstract}
Objectives: As Mycoplasma genitalium is associated with pelvic inflammatory disease (PID), we examined the efficacy of a commonly used PID antimicrobial in treating M genitalium upper genital tract infection.

Methods: In the PID Evaluation and Clinical Health study of inpatient versus outpatient treatment, 682 women treated with cefoxitin and doxycycline for clinically suspected PID had stored cervical and endometrial specimens available for analysis. In the current sub study, we compared baseline endometritis, short term treatment failure (continued endometritis and pelvic pain 30 days following treatment) and sequelae among women with and without $M$ genitalium, identified using PCR.
\end{abstract}

Results: Endometrial $M$ genitalium was associated with baseline endometritis (adjusted OR 3.0, 95\% Cl 1.5 to 6.1). Among women with a positive baseline M genitalium test, $41 \%$ tested positive again 30 days following treatment. Women testing positive compared to those testing negative for $M$ genitalium at baseline had an increased risk of short-term treatment failure (RR 4.6, $95 \% \mathrm{Cl} 1.1$ to 20.1). Rates of sequelae, including infertility $(22 \%)$, recurrent PID $(31 \%)$ and chronic pelvic pain $(42 \%)$, were high among women testing positive for endometrial $M$ genitalium at baseline. There was a non-significant trend towards increased infertility, chronic pelvic pain and recurrent PID, and decreased pregnancy and live birth following $M$ genitalium infection.

Conclusions: $M$ genitalium is associated with endometritis and short-term PID treatment failure. Cefoxitin and doxycycline, a Centers for Disease Control and Prevention recommended PID treatment regimen, is ineffective for the treatment of $M$ genitalium upper genital tract infection.

Mycoplasma genitalium was first identified in the early 1980s among men with non-gonococcal urethritis. ${ }^{1}$ Because this pathogen is extremely difficult to culture, only with polymerase chain reaction (PCR) technology has research into $M$ genitalium pathogenicity progressed. Numerous studies have confirmed the role of $M$ genitalium in drug resistant non-gonococcal urethritis, ${ }^{2}{ }^{3}$ and some have also linked $M$ genitalium with cervicitis $^{45}$ independent of Chlamydia trachomatis and Neisseria gonorrhoeae. Cervicitis is considered a risk factor for progression to pelvic inflammatory disease (PID) - the common infection and inflammation of the female upper genital tract, which may lead to major reproductive morbidity including infertility. ${ }^{6}$
Although PID has polymicrobial aetiology, with $C$ trachomatis and/or $N$ gonorrhoeae accounting for approximately a third to a half of cases, ${ }^{78}$ up to $70 \%$ of PID cases have an unidentified aetiology. Bacterial vaginosis-associated organisms have been associated with PID, ${ }^{10}$ and because $M$ genitalium is associated with cervicitis ${ }^{4}$ it is reasonable to hypothesise that it also causes PID. Indeed, this organism induces salpingitis in monkeys, ${ }^{11}$ causes morphological changes in ciliated fallopian tube cells ${ }^{12}$ and has been detected in fallopian tube tissue in a woman with salpingitis. ${ }^{13}$ Whereas studies have associated $M$ genitalium with PID and endometritis, ${ }^{14-16}$ the efficacy of commonly used PID antimicrobials in treating $M$ genitalium upper genital tract infection is unknown.

\section{MATERIALS AND METHODS \\ Patient population}

Women who participated in the PID Evaluation and Clinical Health (PEACH) Study and had previously collected and stored cervical and endometrial specimens were studied. The PEACH Study methods have been described in detail elsewhere. Briefly, women aged 14 to 37 years were recruited between March 1996 and February 1999 from emergency departments, obstetrics and gynaecology clinics, sexually transmitted disease (STD) clinics and private practices at 13 US clinical sites. Altogether, 831 women with clinically suspected PID, defined by pelvic pain of less than 30 days duration, pelvic organ tenderness and leukorrhea, mucopurulent cervicitis or untreated cervicitis were enrolled. For this subsequent $M$ genitalium study, stored endometrial and cervical specimens were available for 611 and 682 of participants, respectively.

\section{Randomisation and treatment}

Patients in the parent PEACH study were randomised to inpatient or outpatient antibiotic treatment. The inpatient regimen consisted of cefoxitin $(2 \mathrm{~g})$ parenterally every 6 hours and doxycycline (100 mg) orally twice a day for a total of 14 days. The outpatient regimen consisted of a single dose of cefoxitin $(2 \mathrm{~g})$ intramuscularly plus probenecid $(1 \mathrm{~g})$ orally, then doxycycline $(100 \mathrm{mg})$ to be taken orally twice a day for 14 days. As treatment modality was not associated with reproductive morbidities in the PEACH study, ${ }^{7}$ we do not distinguish between inpatient and outpatient regimens or include them as a covariate in these analyses. 


\section{M genitalium PCR}

Previously collected cervical and endometrial specimens stored at $-70^{\circ} \mathrm{C}$ were tested for $M$ genitalium using the MgPa-IMW PCR assay targeting the $\mathrm{MgPa}$ gene ${ }^{17}$ by technicians masked to endometrial histology, treatment failure and reproductive sequelae. This assay has an analytical sensitivity of 15 genomes ${ }^{17}$ and a high clinical sensitivity and specificity relative to transcription-mediated amplification (TMA) - another $M$ genitalium NAAT assay..$^{18}$ For all samples testing positive, a second $\mathrm{MgPa}$ PCR assay was performed using another aliquot of the sample to rule out PCR product contamination or crosscontamination; all samples initially positive were verified as positive in this confirmatory test.

\section{Endometrial biopsies and microbiological studies}

At baseline and 30 days in the parent PEACH study, an endometrial biopsy was obtained for histology, chlamydial PCR (Roche Diagnostics, USA), and gonococcal culture. In a subset of 352 women (240 women in the current sub study), endometrial specimens were cultured for Mycoplasma hominis and Ureaplasma urealyticum. ${ }^{19}$ Endometrial histology was determined based on haematoxylin and eosin stained and methyl green pyronine stained biopsy tissue specimens, read separately by two reference pathologists who reviewed the slide together to reach consensus upon disagreement. A modification ${ }^{20}$ of the criteria proposed by Kiviat et $a l^{8}$ was used to define endometritis. A classification of endometritis was given upon finding at least five neutrophils in the endometrial surface epithelium in the absence of menstrual endometrium and/or at least two plasma cells in the endometrial stroma. Cervical swabs were used for $N$ gonorhoeae cultures and $C$ trachomatis PCR assays. Vaginal smears were Gram stained for bacterial vaginosis as described by Nugent et al. ${ }^{10}$

\section{Follow up}

Participants were monitored with in-person visits at 5 and 30 days. Subsequent telephone interviews were conducted every 3 months during the first year and then every 4 months until June 2004, at which point we had follow up information for 541 women, representing a mean follow up of 84 months. Reproductive outcomes included the occurrence of a first pregnancy, first live birth, infertility, chronic pelvic pain and recurrent PID. Infertility was defined when a sexually active woman with at least 12 months of follow up did not report conception (positive urine or blood test or doctor's diagnosis of pregnancy) despite rare or no use of a contraceptive method. Chronic pelvic pain was defined as pain reported during at least two consecutive follow up interviews; thereby, suggesting a minimum of 6 months duration. Recurrent PID was self reported and verified whenever medical records were available (in $45 \%$ of the cohort). Recurrent PID was confirmed in $76 \%$ of medical records and rates by self report and medical record review were similar. ${ }^{7}$ As few women experienced an ectopic pregnancy during follow up $(n=6)$, we did not include this as an outcome.

\section{Statistical methods}

Baseline descriptive characteristics were compared between women with and without $M$ genitalium using $\chi^{2}$ tests of proportions. The cross-sectional association between $M$ genitalium and endometritis at baseline was additionally determined using a logistic model, fit with $M$ genitalium, age, race, $N$ gonorrhoeae and $C$ trachomatis as explanatory variables.
Relative risks were calculated for the prospectively determined outcomes. Incident endometritis was defined by a negative histological test for endometritis at baseline followed by a positive histological categorisation for endometritis 30 days following treatment. To examine treatment failure, we categorised persistent endometritis as histological endometritis identified at both the baseline and 30 day examinations. Logbinomial regressions determined the relative risks and $95 \%$ confidence intervals (CIs) for endometritis at 30 days, incident endometritis, persistent endometritis, recurrent PID, infertility, chronic pelvic pain, pregnancy and live birth, and included $M$ genitalium, age and race as explanatory variables. Infertility, pregnancy and live birth models were additionally adjusted for self reported infertility at baseline, and models predicting treatment failure were additionally adjusted for intercourse between baseline and 30 days and self reported partner treatment. Analyses were conducted first using both cervical and endometrial $M$ genitalium PCR results combined and were then repeated using only endometrial PCR. All analyses were conducted in the entire cohort and in a subset of women with negative tests for $N$ gonorrhoeae or $C$ trachomatis. SAS version 9.1 for Windows was used for all statistical comparisons.

\section{RESULTS}

We detected $M$ genitalium in 88 (15\%) of women at baseline. Altogether, $11 \%$ of cervical specimens and $8 \%$ of endometrial specimens were positive for $M$ genitalium; infections at these sites were highly correlated (Phi correlation 0.63, p<0.0001), with $74 \%$ of endometrial positive cases also positive at the cervix and $60 \%$ of cervical positive cases also positive in the endometrium.

Women who tested positive compared to those who tested negative for $M$ genitalium were more than twice as likely to have histologically confirmed endometritis at baseline (odds ratio (OR) 2.6, 95\% CI 1.5 to 4.6; table 1). After adjustment for age, race, $N$ gonorrhoeae and $C$ trachomatis, women testing positive for $M$ genitalium were two times more likely to have baseline endometritis (OR 2.0, 95\% CI 1.0 to 4.2). Participants who tested positive and negative for $M$ genitalium had similar scores for self rated pelvic pain at baseline. However, whereas women who tested positive for $M$ genitalium alone scored their pelvic pain similarly to those who tested positive for $C$ trachomatis alone ( $p=0.5)$, women testing positive for $M$ genitalium alone had self rated pelvic pain scores, which were significantly lower than those among women testing positive for $N$ gonorrhoeae alone at baseline (58.0 (SD 21.9) vs 72.3 (SD 23.9), $p=0.01$ ). Women with versus those without $M$ genitalium identified in endometrial and/or cervical specimens were more likely to be less than 25 years of age, have concurrent $N$ gonorrhoeae and $C$ trachomatis, and report vaginal douching, drug use and cigarette smoking (table 1).

Overall, 23 out of 56 women (41\%) with $M$ genitalium identified in either the cervix and/or endometrium at baseline had $M$ genitalium persistently identified 30 days following treatment. Of women with endometrial specimens obtained at both baseline and 30 days, 44\% (14/32) had persistent endometrial $M$ genitalium detected. Women with $M$ genitalium identified in the endometrium were 4.5 times more likely to experience short-term treatment failure, defined by histological identification of endometritis and persistent pelvic pain at the 30 day follow up clinic visit (adjusted rate ratio (RR) 4.6, 95\% CI 1.1 to 20.1; table 2). Having $M$ genitalium identified in the endometrium at baseline was associated with both an increased risk of incident endometritis at 30 days (adjusted RR 5.0, 
Table 1 Characteristics of women with and without Mycoplasma genitalium identified in the endometrium and/or cervix

\begin{tabular}{|c|c|c|c|}
\hline Characteristic & $\begin{array}{l}\mathrm{Mg}+\mathbf{n}=\mathbf{8 8} \\
\mathrm{n}(\%)\end{array}$ & $\begin{array}{l}\mathrm{Mg}-\mathrm{n}=498 \\
\mathrm{n}(\%)\end{array}$ & p Value \\
\hline Histological endometritis* & $43 / 63(68.3)$ & $197 / 439(44.9)$ & 0.0005 \\
\hline Pelvic pain score $†$ (mean SD) & 67.7 (SD 21.3) & 65.6 (SD 21.5) & 0.392 \\
\hline \multicolumn{4}{|l|}{ Co-infection } \\
\hline $\begin{array}{l}N \text { gonorrhoeae and/or } \\
\text { C trachomatis }\end{array}$ & $43 / 65(66.2)$ & $172 / 435(39.5)$ & $<0.0001$ \\
\hline$N$ gonorrhoeae alone & $26 / 59(44.1)$ & $111 / 423(26.2)$ & 0.0045 \\
\hline C trachomatis alone & $23 / 74(31.1)$ & $91 / 484(18.8)$ & 0.0147 \\
\hline M hominis & 4/37 (10.8) & $17 / 203(8.4)$ & 0.6295 \\
\hline$U$ urealyticum & $1 / 37(2.7)$ & $15 / 203(7.4)$ & 0.2932 \\
\hline BV & $51 / 80(63.8)$ & $254 / 453(56.1)$ & 0.2006 \\
\hline \multicolumn{4}{|l|}{ Demographic } \\
\hline \multicolumn{4}{|l|}{ Age } \\
\hline$<25$ years & $71 / 88(80.7)$ & $319 / 498(64.1)$ & \\
\hline$\geqslant 25$ years & $17 / 88(19.3)$ & $179 / 498(35.9)$ & 0.0023 \\
\hline \multicolumn{4}{|l|}{ Race/ethnicity } \\
\hline African American & 69/88 (78.4) & $358 / 498$ (71.9) & \\
\hline White/Hispanic/Other & $19 / 88(21.6)$ & $140 / 498(28.1)$ & 0.2047 \\
\hline \multicolumn{4}{|l|}{ Marital status } \\
\hline Unmarried & $71 / 78(91.0)$ & $411 / 466(88.2)$ & \\
\hline Married & $7 / 78(9.0)$ & $55 / 466(11.8)$ & 0.4669 \\
\hline \multicolumn{4}{|l|}{ Education } \\
\hline$<$ High school & $38 / 88(43.2)$ & 189/497 (38.0) & \\
\hline$\geqslant$ High school & $50 / 88(56.8)$ & $308 / 497(62.0)$ & 0.3605 \\
\hline \multicolumn{4}{|l|}{ Sexual history } \\
\hline History of STD: & $51 / 86(59.3)$ & $288 / 485(59.4)$ & 0.9890 \\
\hline History of BV & $13 / 86(15.1)$ & $117 / 482(24.3)$ & 0.0626 \\
\hline Sexually active & $78 / 88(88.6)$ & 415/498 (83.3) & 0.2095 \\
\hline $\begin{array}{l}\text { Rare/occasional condom } \\
\text { use§ }\end{array}$ & $60 / 78(76.9)$ & 289/415 (69.6) & 0.1943 \\
\hline Consistent condom use & $7 / 78(9.0)$ & $57 / 415(13.7)$ & 0.2511 \\
\hline$\geqslant 2$ sexual partners & 10/88 (11.4) & $46 / 498(9.2)$ & 0.5316 \\
\hline \multicolumn{4}{|l|}{ Behavioural } \\
\hline $\begin{array}{l}\text { Vaginal douche, } 2+\text { times } \\
\text { in past month }\end{array}$ & $26 / 88(29.6)$ & $88 / 498(17.7)$ & 0.0095 \\
\hline Drug use & $32 / 88$ (36.4) & 125/495 (25.3) & 0.0304 \\
\hline Current smoker & 49/88 (55.7) & $194 / 495$ (39.2) & 0.0038 \\
\hline
\end{tabular}

$* \geqslant 5$ surface epithelium neutrophils per $\times 400$ field absent of menstrual endometrium and/or $\geqslant 2$ stromal plasma cells per $\times 120$ field; †mean composite pain score $=$ (mean of current pelvic pain score, average pelvic pain score and worst pelvic pain score) $\times 10$; thistory of Neisseria gonorrhoeae, Chlamydia trachomatis or

Trichonomas vaginalis; $\S$ condoms used 0 to 5 out of 10 sexual encounters; 9 condoms used in 10 out of 10 sexual encounters.

$\mathrm{BV}$, bacterial vaginosis; STD, sexually transmitted disease.

95\% CI 1.2 to 20.6) and persistent endometritis, identified histologically at both baseline and 30 days following treatment (adjusted RR 4.1, 95\% CI 1.4 to 11.9). In models additionally adjusted for $N$ gonorrhoeae and $C$ trachomatis, endometrial $M$ genitalium was associated with incident endometritis (adjusted RR 6.0, 95\% CI 1.4 to 27.1) and treatment failure (adjusted RR 5.2, 95\% CI 0.9 to 30.6), although the association with treatment failure was of borderline statistical significance. Results were similar among a subset of women testing negative for $C$ trachomatis and $N$ gonorrhoeae, although cell sizes were small and confidence intervals large. In this group of women without $N$ gonorrhoeae or $C$ trachomatis, those with $M$ genitalium identified in the endometrium at baseline were nearly nine times as likely to be categorised as having endometritis at the 30 day examination (adjusted RR 8.8, 95\% CI 2.0 to 39.6) and over 13 times as likely to be defined as having incident endometritis at 30 days (adjusted RR 13.4, 2.4 to 75.2). As co-infection was common in this study, models with microbiological interaction terms were additionally examined. $M$ genitalium did not significantly interact with $N$ gonorrhoeae or $C$ trachomatis to additionally influence the risk of incident endometritis or treatment failure. In models adjusting for $M$ hominis and $U$ urealyticum, $M$ genitalium was associated with almost a 2.5 fold increased risk of persistent endometritis (RR 2.4, 95\% CI 0.5 to 12.4). However, as $M$ hominis and $U$ urealyticum were only cultured on a subset of women in the parent PEACH study, a smaller sample size resulted in a wide confidence interval.

Rates of infertility (22\%), recurrent PID (31\%) and chronic pelvic pain $(42 \%)$ were high among women testing positive for endometrial $M$ genitalium at baseline. Although the rates of reproductive morbidity were not significantly higher among women testing positive for $M$ genitalium, all relationships were in the hypothesised direction (table 3). Women with versus those without endometrial $M$ genitalium experienced modest, non-significantly elevated risks of recurrent PID, infertility and chronic pelvic pain, and were slightly but non-significantly less likely to experience a pregnancy or live birth.

\section{DISCUSSION}

We detected $M$ genitalium at a high prevalence (15\%) among an urban US population of women with non-gonococcal, nonchlamydial PID, similar to that found in prior PID studies among Kenyan $(16 \%)^{16}$ and UK women $(13 \%) .{ }^{15}$ In the PEACH study, about $14 \%$ of women were infected with $C$ trachomatis, $15 \%$ were infected with $N$ gonorrhoeae and $5 \%$ were co-infected with these two pathogens. ${ }^{7}$ Thus, $M$ genitalium was as prevalent as $C$ trachomatis or $N$ gonorrhoeae among this population of women with PID.

Women with endometrial $M$ genitalium were over three times more likely to have histologically confirmed endometritis compared to women without $M$ genitalium identified at this site. Our study suggests that the relationship between $M$ genitalium and endometritis is independent and causal, since among women without concurrent $N$ gonorrhoeae and/or $C$ trachomatis, endometrial $M$ genitalium was associated with over a 13-fold risk of incident endometritis at the 30 day follow up visit. Our results are consistent with previous cross-sectional studies associating $M$ genitalium with PID. ${ }^{14}{ }^{16}$ Further, we found that although pelvic pain was similar between women testing positive and negative for $M$ genitalium, women who tested positive for $M$ genitalium alone reported significantly less pelvic pain than women who tested positive for $N$ gonorrhoeae alone. Pelvic pain scores were lower and similar among women who tested positive for $M$ genitalium alone and $C$ trachomatis alone, suggesting that, like $C$ trachomatis, $M$ genitalium may also play a role in asymptomatic or "silent" PID.

To our knowledge, ours is the first study to investigate treatment failure among PID patients with $M$ genitalium identified in the endometrium. Persistence of $M$ genitalium following treatment with a standard Centers for Disease Control and Prevention recommended PID treatment regimen was very high: $44 \%$ of women with baseline endometrial positive specimens tested positive again 30 days following treatment. In contrast, only $2-4 \%$ of women in the PEACH study had persistent or recurrent gonococcal or chlamydial cervicitis when retested at 30 days. $^{7}$ Women with $M$ genitalium identified in the endometrium at baseline were four times as likely to experience persistent endometritis and approximately 4.5 times as likely to experience treatment failure-defined as the presence of both endometritis and pelvic pain 30 days following treatment for PID. 
Table 2 Endometritis 30 days following treatment with cefoxitin and doxycycline and short-term treatment failure (endometritis at 30 days and persistent pelvic pain) among women with and without baseline Mycoplasma genitalium (MG) genital tract infection

\begin{tabular}{|c|c|c|c|c|c|c|}
\hline Outcome & $\begin{array}{l}\text { Cervical and/or } \\
\text { endometrial } \mathbf{M g}+\mathbf{n} \\
(\%) \mathbf{n}=\mathbf{8 8}\end{array}$ & $\begin{array}{l}\text { Cervical and/or } \\
\text { endometrial } \mathrm{Mg}-\mathrm{n} \\
(\%) \mathrm{n}=498\end{array}$ & $\begin{array}{l}\text { Adjusted RR- } \\
\text { (95\% CI) }\end{array}$ & $\begin{array}{l}\text { Endometrial } \mathbf{M g}+\mathbf{n} \\
(\%) \mathbf{n}=\mathbf{5 0}\end{array}$ & $\begin{array}{l}\text { Endometrial } \mathrm{Mg}-\mathrm{n} \\
(\%) \mathrm{n}=561\end{array}$ & Adjusted RR $\uparrow(95 \%$ CI) \\
\hline Treatment failure ${ }^{*}$ & $8(40.0)$ & $26(17.5)$ & $2.6(0.8$ to 8.9$)$ & $6(50.0)$ & $30(18.5)$ & $4.6(1.1$ to 20.1$)$ \\
\hline Endometritis at 30 days $\dagger$ & $25(56.8)$ & $119(39.0)$ & $1.9(0.9$ to 3.9$)$ & $19(70.4)$ & $131(39.2)$ & $4.0(1.5$ to 10.9$)$ \\
\hline Incident endometritis & $6(24.0)$ & $35(15.8)$ & $1.5(0.4$ to 5.0$)$ & $5(38.5)$ & $37(15.4)$ & $5.0(1.2$ to 20.6$)$ \\
\hline Persistent endometritis§ & $17(47.2)$ & $73(28.2)$ & $2.1(1.0$ to 4.6$)$ & $13(61.9)$ & $82(28.8)$ & $4.1(1.4$ to 11.9$)$ \\
\hline
\end{tabular}

*Treatment failure is defined as both endometritis and pelvic pain present at the 30 day clinic visit; †All women categorised as having endometritis at the 30 day clinic visit; fincludes women without endometritis at baseline who are categorised as having endometritis at the 30 day clinic visit; §includes women who are categorised as having endometritis at both baseline and 30 days; - Adjusted for age and race, self reported partner treatment and intercourse between baseline and the 30 day clinic visit.

Several attributes of $M$ genitalium indicate that it is resistant to cefoxitin and doxycycline. First, mycoplasmal bacteria lack a cell wall and are thus resistant to cell wall inhibiting antibiotics. Second, an $M$ genitalium strain with increased tetracycline resistance has been isolated ${ }^{21}$ and $M$ genitalium is associated with persistent non-gonococcal urethritis among men treated with tetracyclines. ${ }^{22}$ Most women with PID are treated with antibiotics directed toward $N$ gonorrhoeae and/or $C$ trachomatis, despite the fact that these pathogens account for only a third to a half of PID cases. Indeed, in the PEACH study approximately $60 \%$ of women had non-gonococcal, non-chlamydial PID. ${ }^{7}$ Over a third of the women had persistent endometritis post treatment, ${ }^{7}$ identifying a sizeable portion as having ongoing upper genital tract inflammation and demonstrating that antibiotics used to treat this critically important upper tract syndrome are suboptimal. $M$ genitalium has demonstrated variable resistance to fluoroquinolones ${ }^{23}$ and susceptibility to macrolides, although azithromycin resistant strains have recently been identified. ${ }^{24} \mathrm{~A}$ newer quinolone, moxifloxacin, has recently been shown to exhibit a high degree of activity against $M$ genitalium. ${ }^{21}$ Randomised clinical trials assessing these alternative regimens for the treatment of $M$ genitalium and PID are needed.

It is possible that other organisms resistant to doxycycline or cefoxitin may have confounded the relationship between $M$ genitalium and persistent endometritis. However, $M$ genitalium was not associated with endometrial $M$ hominis or $U$ urealyticum - organisms that express tetracycline resistance ${ }^{25}$ and contain tet $\mathrm{M}$ genes that encode tetracycline resistance. ${ }^{26}$ Further, adjustment for these pathogens only slightly attenuated the relationship between $M$ genitalium and persistent endometritis. Additionally, we recognise that PID may recur among women who engage in risky sexual behaviour during treatment, and this may also confound treatment failure comparisons. However, in models adjusted for self reported partner treatment and intercourse, $M$ genitalium was significantly predictive of treatment failure, supporting the idea that $M$ genitalium antibiotic resistence may lead to persistent PID.
Although a positive $M$ genitalium test was not significantly predictive of reproductive morbidity in our study, results were in the hypothesised direction. Infertility, chronic pelvic pain and recurrent PID were more common among women testing positive for endometrial $M$ genitalium, and women who tested positive were less likely to become pregnant or have a live birth. Certainly, these reproductive outcomes are more subject to misclassification and bias than shorter term outcomes due to loss of follow up and reliance on self reported data. Such misclassification may have biased our results towards the null. Additionally, it is possible that women may have had prior $M$ genitalium infection that resulted in tubal damage preceding the baseline PEACH study PID episode. As such prior infection would not be identified using PCR, which identifies acute infection, this may have biased our results towards the null. Indeed, in a study of 308 women undergoing in vitro fertilisation treatment, women determined to have tubal factor infertility were over three times as likely to be seropositive for $M$ genitalium. ${ }^{27}$ Moreover, among a study of 212 couples attending infertility clinics, although $M$ genitalium was not detected by PCR in any cervical swab specimens, $M$ genitalium seropositivity was strongly associated with tubal factor infertility. ${ }^{28}$ This supports the idea that previous $M$ genitalium infection may result in permanent damage and occlusion of fallopian tubes. Lastly, although we found $M$ genitalium to be associated with treatment failure, we have previously reported that short-term markers, including pelvic tenderness at 5 and 30 days, are not predictive of reproductive morbidity in the PEACH study population. ${ }^{29}$ As over two-thirds of women in the PEACH study presented for treatment with 3 or more days of pelvic pain, ${ }^{7}$ and since delayed care for PID is associated with almost a threefold increased risk for infertility, ${ }^{30}$ all women in the PEACH study may have been at increased risk for reproductive sequelae regardless of short-term treatment response. Indeed, women in the PEACH study experienced distressingly high rates of morbidity regardless of microbial aetiology of PID (infertility $17 \%{ }^{7}$ recurrent PID $14 \%^{7}$ and chronic pelvic pain $37 \%{ }^{31}$ ).

Table 3 Sequelae and long-term treatment failure among women with and without baseline Mycoplasma gentalium (MG) genital tract infection

\begin{tabular}{|c|c|c|c|c|c|c|}
\hline Outcome & $\begin{array}{l}\text { Cervical and/or } \\
\text { endometrial } \mathrm{Mg}+\mathrm{n}(\%) \\
\mathrm{n}=\mathbf{8 8}\end{array}$ & $\begin{array}{l}\text { Cervical and/or } \\
\text { endometrial } \mathrm{Mg}-\mathrm{n}(\%) \\
\mathrm{n}=498\end{array}$ & $\begin{array}{l}\text { Adjusted RR* } \\
\text { (95\% CI) }\end{array}$ & $\begin{array}{l}\text { Endometrial } \mathbf{M g}+\mathbf{n}(\%) \\
\mathbf{n}=\mathbf{5 0}\end{array}$ & $\begin{array}{l}\text { Endometrial } \mathrm{Mg}-\mathrm{n}(\%) \\
\mathrm{n}=\mathbf{5 6 1}\end{array}$ & $\begin{array}{l}\text { Adjusted RR* } \\
(95 \% \mathrm{CI})\end{array}$ \\
\hline $\begin{array}{l}\text { Recurrent pelvic } \\
\text { inflammatory disease }\end{array}$ & $24(28.2)$ & $98(20.8)$ & $1.6(0.8$ to 3.1$)$ & $15(30.6)$ & $111(20.8)$ & $1.8(0.8,3.8)$ \\
\hline Infertility & $15(17.4)$ & $95(19.4)$ & $1.4(0.6$ to 2.9$)$ & $11(22.0)$ & $104(18.9)$ & $1.1(0.7$ to 4.0$)$ \\
\hline Chronic pelvic pain & $37(43.5)$ & $198(41.4)$ & $1.6(0.9$ to 2.9$)$ & $21(42.0)$ & $226(42.1)$ & $1.3(0.6$ to 2.7$)$ \\
\hline Pregnancy & $53(61.6)$ & $278(56.9)$ & $0.8(0.4$ to 1.5$)$ & $29(58.0)$ & $317(57.6)$ & 0.7 (0.3 to 1.4$)$ \\
\hline
\end{tabular}

${ }^{*}$ All models are adjusted for age, race, Neisseria gonorrhoeae and Chlamydia trachomatis. Infertility, pregnancy and live birth models are additionally adjusted for self reported infertility at baseline. 


\section{Key messages}

- Mycoplasma genitalium is frequently detected from the cervix and endometrium of women with clinically suspected pelvic inflammatory disease (PID).

- $M$ genitalium is associated with endometritis and short-term PID treatment failure, as evidenced by persistent endometritis and continued pelvic pain.

- Cefoxitin and doxycycline, a Center for Disease Control recommended PID treatment regimen, is ineffective for the treatment of $M$ genitalium upper genital tract infection.

Certainly, women with $M$ genitalium identified in either the cervix or endometrium had high rates of infertility (18\%), recurrent PID (28\%) and chronic pelvic pain (44\%). These infertility rates are approximately 2.5 times higher than that reported from a study utilising 2002 National Survey of Family Growth data, ${ }^{32}$ suggesting that preservation of fertility may be suboptimal for many women treated for PID.

Recommended PID treatment regimens are based on clinical trial data demonstrating overall high rates of clinical cure. PID is well recognised though as having a polymicrobial aetiology, and not all microbial infections may respond equally well to the same antimicrobial. In our study of women with clinically suspected PID, we found $M$ genitalium to be associated with endometritis and short-term PID treatment failure, as evidenced by persistent endometritis and continued pelvic pain. We conclude that cefoxitin and doxycycline, a Center for Disease Control recommended PID treatment regimen, is ineffective for the treatment of $M$ genitalium upper genital tract infection.

Acknowledgements: Investigators involved in the PEACH Study include A Amortegui; S L Hendrix; S L Hillier; R L Holley; D B Nelson; J Nichols Jr; J Peipert; H Randall; D Schubeck; S J Sondheimer; D E Soper; R L Sweet; W Trout; G Trucco; H C Wiesenfeld and T Yalcinkaya. We thank and acknowledge the efforts of interviewers who recruited and interviewed study participants: S Allen, A Baum, C Becker, $\mathrm{H}$ Cohen-Webb, A Cooper, P Crowner, L Curll, J Faas, A Farmer, E Hoffman, A Holdredge, A Howell, S Kay, F Leboeuf, IMacio, K Martin, M McNamee, A Meers, K Miller, A Montagno, J Mowery, J Mitton, C Myers, B Nobels, T Pealer, A Rideout, G Rothstein, C Sams, T Schuda, B Schiavoni, M Scott, K Timbers, S Whitaker, L Williams and M Vincetic. We also gratefully acknowledge consultants T Songer, J Lave and S Kelsey, and technical assistant D Bass.

Funding: HS08358-05 from the Agency for Healthcare Research and Quality Development and 1 R01 Al067661-01A2 from the National Institute of Allergy and Infectious Diseases.

\section{Competing interests: None.}

Contributors: Study concept and design: CLH, PAT, RBN; acquisition of data: CLH, PAT, RBN; analysis and interpretation of data: CLH, PAT, SGA; drafting of manuscript: CLH; critical revision of manuscript for important intellectual content: PAT, SFK, RBN; statistical analysis: SL, SLH; obtained funding: CLH; supervision CLH.

\section{REFERENCES}

1. Tully JG, Taylor-Robinson D, Cole RM, et al. A newly discovered mycoplasma in the human urogenital tract. Lancet 1981;1288-91.

2. Totten PA, Schwartz MA, Sjostrom KE, et al. Association of Mycoplasma genitalium with nongonococcal urethritis in heterosexual men. J Infect Dis 2001;183:269-76.

3. Jensen JS. Mycoplasma genitalium: a cause of non-gonococcal urethritis? Genitourin Med 1994;70:363.

4. Manhart LE, Critchlow CW, Holmes KK, et al. Mucopurulent cervicitis and Mycoplasma genitalium. J Infect Dis 2003;187:650-57.
5. Pepin J, Labbe AC, Khonde N, et al. Mycoplasma genitalium: an organism commonly associated with cervicitis among west African sex workers. Sex Transm Infect 2005;81:67-72

6. Westrom L. Effect of acute pelvic inflammatory disease on fertility. Am J Obstet Gynecol 1975;121:707-13

7. Ness RB, Soper DE, Holley RL, et al. Effectiveness of inpatient and outpatient treatment strategies for women with pelvic inflammatory disease: results from the PID Evaluation and Clinical Health (PEACH) randomized trial. Am J Obstet Gynecol 2002; 186:929-37

8. Kiviat NB, Wolner-Hanssen P, Eschenbach DA, et al. Endometrial histopathology in patients with culture-proven upper genital tract infection and laparoscopically diagnosed acute salpingitis. Am J Surg Pathol 1990;14(2):167-75.

9. Haggerty CL, Hillier SL, Bass DC, et al. Bacterial vaginosis and anaerobic bacteria are associated with endometritis. Clin Infect Dis 2004;39:990-5.

10. Nugent RP, Krohn MA, Hillier SL. Reliability of diagnosing bacterial vaginosis is improved by a standardized Gram stain interpretation. J Clin Microbiol 1991;29:297-301.

11. Møller BR, Taylor-Robinson D, Furr PM, et al. Acute upper genital-tract disease in female monkeys provoked experimentally by Mycoplasma genitalium. Br J Exp Pathol 1985;66:417-26.

12. Baczynska A, Funch P, Fedder J, et al. Morphology of human fallopian tubes after infection with Mycoplasma genitalium and Mycoplasma hominis - in vitro organ culture study. Hum Reprod 2007;22:968-79.

13. Cohen CR, Mugo NR, Astete SG, et al. Detection of Mycoplasma genitalium in women with laparoscopically diagnosed acute salpingitis. Sex Transm Infect 2005;81:463-6.

14. Uno M, Deguchi $\mathrm{T}$, Komeda $\mathrm{H}$, et al. Mycoplasma genitalium in the cervices of Japanese women. Sex Transm Dis 1997;24:284-6.

15. Simms I, Eastick K, Mallinson H, et al. Associations between Mycoplasma genitalium, Chlamydia trachomatis and pelvic inflammatory disease. J Clin Pathol 2003;56:616-18.

16. Cohen CR, Manhart LE, Bukusi EA, et al. Association between Mycoplasma genitalium and acute endometritis. Lancet 2002;359:765-6.

17. Dutro SM, Hebb JK, Garin CA, et al. Development and performance of a microwellplate-based polymerase chain reaction assay for Mycoplasma genitalium. Sex Transm Dis 2003;30:756-63.

18. Wroblewski JK, Manhart LE, Dickey KA, et al. Comparison of transcriptionmediated amplification and PCR assay results for various genital specimen types for detection of Mycoplasma genitalium. J Clin Microbiol 2006;44:3306-12.

19. Hillier SL, Krohn MA, Rabe LK, et al. The normal vaginal flora, H2O2-producing lactobacilli, and bacterial vaginosis in pregnant women. Clin Infect Dis 1993; 16:S273-81

20. Ness RB, Keder LM, Soper DE, et al. Oral contraception and the recognition of endometritis. Am J Obstet Gynecol 1997;176:580-5.

21. Hamasuna R, Osada $Y$, Jensen JS, et al. Antibiotic susceptibility testing of Mycoplasma genitalium by TaqMan 5' nuclease real-time PCR. Antimicrob Agents Chemother 2005;49:4993-8.

22. Horner P, Thomas B, Gilroy CB, et al. Role of Mycoplasma genitalium and Ureaplasma urealyticum in acute and chronic nongonococcal urethritis. Clin Infect Dis 2001;32:995-1003

23. Horner PJ, Thomas B, Gilroy CB, et al. Do all men attending departments of genitourinary medicine need to be screened for non-gonococcal urethritis? Int J STD AIDS 2002;13:667-73

24. Bradshaw CS, Jensen JS, Tabrizi SN, et al. Azithromycin failure in Mycoplasma genitalium urethritis. Emerg Infect Dis 2006;12:1149-52.

25. Ullmann U, Schubert S, Krausse R. Comparative in-vitro activity of levofloxacin, other fluoroquinolones, doxycycline and erythromycin against Ureaplasma urealyticum and Mycoplasma hominis. J Antimicrob Chemother 1999;43:33-6.

26. Samra Z, Rosenberg S, Soffer Y. In vitro susceptibility of Mycoplasma hominis clinical isolates to tetracyclines, quinolones and macrolides. Diagn Microbiol Infect Dis 2002;44:359-61.

27. Clausen HF, Fedder J, Drasbek M, et al. Serological investigation of Mycoplasma genitalium in infertile women. Hum Reprod 2001;16:1866-74.

28. Svenstrup HF, Fedder J, Kristoffersen SE, et al. Mycoplasma genitalium, Chlamydia trachomatis, and tubal factor infertility. Fertil Steril 2008.

29. Trautmann GM, Kip KE, Richter HE, et al. Do short-term markers of treatment efficacy predict long-term sequelae of pelvic inflammatory disease? Am J Obstet Gynecol 2008;198:30-7.

30. Hillis SD, Joesoef R, Marchbanks PA, et al. Delayed care of pelvic inflammatory disease as a risk factor for impaired fertility. Am J Obstet Gynecol 1993; 168:1503-9.

31. Haggerty CL, Ness RB, Amortegui $A$, et al. Endometritis does not predict reproductive morbidity following pelvic inflammatory disease. Am J Obstet Gynecol 2003:188:140-7.

32. Stephen EH, Chandra A, Stephen EH, et al. Declining estimates of infertility in the United States: 1982-2002. Fertil Steril 2006;86:516-23. 